\title{
Statistical tool combined with image analysis to characterize hydrodynamics and mass transfer in a bubble column
}

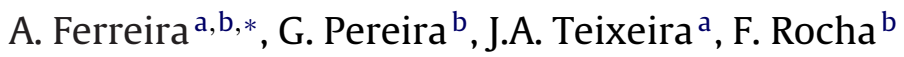 \\ a IBB-Institute for Biotechnology and Bioengineering, Centre of Biological Engineering, Universidade do Minho, Campus de Gualtar, 4710-057 Braga, Portugal \\ ${ }^{\mathrm{b}}$ LEPAE-Laboratory for Process, Environmental and Energy Engineering, Departamento de Engenharia Química, Faculdade de Engenharia, Universidade do Porto, Rua Dr. Roberto \\ Frias s/n, 4200-465 Porto, Portugal
}

\section{A R T I C L E I N F O}

\section{Article history:}

Received 23 June 2011

Received in revised form

23 September 2011

Accepted 28 September 2011

\section{Keywords:}

Bubble column

Mass transfer

Multiphase reactors

Hydrodynamics

Image analysis

\begin{abstract}
A B S T R A C T
Image analysis technique has been proved to be very effective in the quantification of particles size and morphology distributions in different work areas. In the present work this technique was combined with the discriminant factorial analysis (DFA) in order to allow the automatic identification of single bubbles (isolated bubbles without influence of surrounded bubbles) in multiphase systems. With the previous methodology it has been possible to distinguish online and automatically among three different classes of bubbles (single bubbles and medium complexity and large complexity bubbles groups), allowing for the first time the computation of the local bubble population complexity in the system. The automatic and correct characterization of the single bubbles allowed the correct determination of bubble size and, consequently, the specific interfacial area $a$ at different experimental conditions. Agreement between automated and manual classification, measured in terms of a performance index, is $98 \%$ for single bubbles identification. Further, the present work describes the application of such methodology to the study of temperature, type of gas sparger, and liquid phase properties (viscosity and surface tension) influence on the individual components of volumetric liquid side mass transfer coefficient, $k_{L} a$. The results show that the different experimental parameters and liquid properties act by a particular way on $k_{L}$ and $a$.
\end{abstract}

(c) 2011 Elsevier B.V. All rights reserved.

\section{Introduction}

Gas/liquid contactors are used in chemical, biochemical, pharmaceutical, petrochemical and others industries. Bubble column reactors are intensively used as multiphase contactors. Their popularity is essentially related to the simple design and excellent heat and mass transfer properties. The mass transfer from the gas to the liquid is the most important goal of the process in bubble columns reactors. Thus, the characterization of the individual parameters of volumetric liquid side mass transfer coefficient, $k_{L} a$, at different experimental conditions is an imperative process for the correct characterization of the mass transfer process. The liquid-side mass transfer coefficient $k_{L}$ can be estimated from correlations [1] or determined, indirectly, when the interfacial area $a$ and $k_{L} a$ are known. The experimental determination of $a$ can be done using chemical and physical methods. The chemical techniques are based on a reaction of known kinetics in which the absorption rate is a function of the interfacial gas-liquid area [1-7], while

\footnotetext{
* Corresponding author at: IBB-Institute for Biotechnology and Bioengineering, Centre of Biological Engineering, Universidade do Minho, Campus de Gualtar, 4710057 Braga, Portugal. Tel.: +351 225081678; fax: +351225081632.

E-mail address: amaf@fe.up.pt (A. Ferreira).
}

the physical methods are based on the measurement of a physical property. The physical techniques are usually divided in noninvasive methods that give global, cross-section-averaged or local data, and the intrusive probes adequate to local measurements. An extensive overview of the instrumentation techniques developed for multiphase flow analysis in gas/liquid and gas/liquid/solid reactors can be found in Boyer et al. [8].

Despite all the techniques, the correct determination of $a$ remains a challenge, as the bubble size distribution in the column is not constant, due to bubble-bubble and bubble-particle interactions that can lead to breakage or coalescence [9-13]. Moreover, the bubbles are, usually, not spherical and can vary widely in shape, affecting the reliability of some techniques. The non-invasive technique able to obtain, directly, the bubble size and shape is the one based on image analysis technique. The most common limitations of this technique are associated to problems related to wall transparency, out-of-focus, system illumination and image analysis. Most of these limitations can be experimentally solved, however, in what concerns the image analysis the automatic and correct identification of isolated and overlapping bubbles is one of the biggest challenges on this field. Several works can be found in the literature regarding the image analysis from bubble columns [12,14-16]. However, the automatic identification of isolated and overlapping bubbles remains unsolved. The commercial softwares used by the 


\begin{tabular}{|c|c|}
\hline \multicolumn{2}{|c|}{ Nomenclature } \\
\hline$A_{\text {proj }}$ & projected bubble area $\left(\mathrm{m}^{2}\right)$ \\
\hline$a^{2}$ & gas-liquid interfacial area $\left(\mathrm{m}^{-1}\right)$ \\
\hline$a^{*}$ & gas-liquid interfacial area for pure system $\left(\mathrm{m}^{-1}\right)$ \\
\hline$B_{C D}$ & mean complexity degree of the entire population \\
\hline$B_{C D i}$ & complexity degree of the bubble $i$ \\
\hline$C$ & circularity, dimensionless \\
\hline C & oxygen concentration in the liquid $(\mathrm{kg} / \mathrm{L})$ \\
\hline$C_{0}$ & oxygen concentration in the liquid at $t=0(\mathrm{~kg} / \mathrm{L})$ \\
\hline$C^{*}$ & oxygen solubility in the liquid $(\mathrm{kg} / \mathrm{L})$ \\
\hline$D_{e q}$ & equivalent diameter $(\mathrm{m})$ \\
\hline$D_{L}$ & diffusivity of gas in the liquid $\left(\mathrm{m}^{2} / \mathrm{s}\right)$ \\
\hline$d_{10}$ & bubble average diameter $(\mathrm{m})$ \\
\hline$d_{32}$ & Sauter mean diameter $(\mathrm{m})$ \\
\hline$F_{\max }$ & maximal Feret diameters (m) \\
\hline$F_{\min }$ & minimal Feret diameters (m) \\
\hline$f$ & $\begin{array}{l}\text { volumetric concentration of the particles, dimen- } \\
\text { sionless }\end{array}$ \\
\hline$g$ & acceleration due to gravity $\left(\mathrm{m} / \mathrm{s}^{2}\right)$ \\
\hline$k_{L}$ & liquid-side mass transfer coefficient $(\mathrm{m} / \mathrm{s})$ \\
\hline$k_{L} a$ & volumetric liquid side mass transfer coefficient $\left(\mathrm{s}^{-1}\right)$ \\
\hline$N_{i}$ & number of internal zones \\
\hline$n$ & number of bubbles, dimensionless \\
\hline$P$ & perimeter $(\mathrm{m})$ \\
\hline PI & performance index \\
\hline$P b_{i, S}$ & probability of bubble being single \\
\hline$P b_{i, M A}$ & $\begin{array}{l}\text { probability of bubble belong to a medium complex- } \\
\text { ity bubbles group }\end{array}$ \\
\hline$P b_{i, V A}$ & $\begin{array}{l}\text { probability of bubble belong to a large complexity } \\
\text { bubbles group }\end{array}$ \\
\hline$S_{\text {in }}$ & surface occupied by the internal zones $\left(\mathrm{m}^{2}\right)$ \\
\hline$T$ & temperature $\left({ }^{\circ} \mathrm{C}\right)$ \\
\hline$t$ & time $(\mathrm{s})$ \\
\hline$u_{G}$ & superficial gas velocity $(\mathrm{m} / \mathrm{s})$ \\
\hline$\varepsilon_{G}$ & gas holdup, dimensionless \\
\hline & angle $\left(^{\circ}\right)$ \\
\hline$\mu^{*}$ & $\begin{array}{l}\text { effective viscosity of the particle-fluid mixture } \\
(\mathrm{kg} /(\mathrm{ms}))\end{array}$ \\
\hline$\mu_{l}$ and & viscosity of liquid phase $(\mathrm{kg} /(\mathrm{ms}))$ \\
\hline & particle robustness \\
\hline$\sigma$ & surface tension $(\mathrm{N} / \mathrm{m})$ \\
\hline
\end{tabular}

different authors conduct to a manual or semi-manual characterization of the individual bubbles by the operator. Some authors [15] used the concavity index as a criterion to distinguish between isolated and overlapping bubbles: overlapping induces concavities in the object and decreases the concavity index. This criterium seems to not produce significant errors on the bubble size determination for low superficial gas velocity (less than $3 \times 10^{-3} \mathrm{~m} / \mathrm{s}$ ). However, for high superficial gas velocities other criteria need to be found, as a result of bubbles number increase.
A combination of image analysis technique with the discriminant factorial analysis has been proved to be very effective in the quantification of particles size and morphology distributions in crystallization area, as off-line [17] and online [18] techniques.

In the present work, the online technique, developed by Ferreira et al. [18] for characterization of sucrose crystal morphology, was improved in order to allow the automatic identification of single bubbles (isolated bubbles without influence of surrounded bubbles) and bubble groups (medium complexity and large complexity groups) in the bubble column. As this automatic classification is based on several probabilities of each bubble belonging to each of the groups considered $[17,18]$, it was also possible to obtain the complexity or turbulence of the system. This information shows to be very useful in understanding the bubble size distribution and the mass transfer process in the bubble column, mainly in what concerns the influence of the other bubbles on the concentration profiles surrounding individual bubbles, a question that has been recently studied $[19,20]$. The methodology and image analysis development on the present work aims to open new insights for a better understanding of mass transfer phenomena in the bubble column. The developed tool was applied to the study of the influence of temperature, type of gas sparger, and liquid phase properties (viscosity and surface tension) on the individual parameters of volumetric liquid side mass transfer coefficient, $k_{L} a$.

\section{Experimental}

\subsection{Mass transfer experiments}

\subsubsection{Set-up}

The contact device used to perform the mass transfer experiments was the bubble column represented in Fig. 1 with the respective dimensions. The device is a perspex cylindrical column covered by a perspex rectangular box to control the temperature through water circulation. At the bottom a gas chamber is located, where the gas enters first and then passes through a steel sparger, with a thickness of $2 \mathrm{~mm}$, where the bubbles are formed. The sparger has a relative free area of $0.02 \%$ and $0.05 \%$ for an orifice diameter of 0.3 and $0.5 \mathrm{~mm}$, respectively.

\subsubsection{Methodology}

Oxygen mass transfer experiments were performed in twophase system at different temperatures $\left(20,25\right.$ and $\left.30^{\circ} \mathrm{C}\right)$, superficial gas velocities (up to $14 \mathrm{~mm} / \mathrm{s}$ ), gas spargers (orifice diameter of 0.3 and $0.5 \mathrm{~mm}$ ) and liquids (aqueous solutions of alcohol, ethanol (up to $3.43 \mathrm{M}$ ), sodium chloride, $\mathrm{NaCl}$ (up to $3.54 \mathrm{M}$ ) and sucrose (up to $1.41 \mathrm{M}$ )). Air was used as gas phase. The liquid height was $h_{0}=0.32 \mathrm{~m}$ for all experiments (no liquid throughput). Table 1 presents a summary of the experimental conditions used in the present work.

Initially the liquid is deoxygenated by bubbling nitrogen. When the dissolved oxygen concentration is practically zero, humidified air is fed into the column. At this moment the oxygen transfer process from bubbles to the liquid begins and continues

Table 1

Experimental conditions used in the present work.

\begin{tabular}{|c|c|c|c|c|}
\hline \multicolumn{3}{|l|}{ Liquid phase } & \multirow{2}{*}{$\begin{array}{l}\text { Gas phase } \\
u_{G} \times 10^{3}(\mathrm{~m} / \mathrm{s})\end{array}$} & \multirow{2}{*}{$\begin{array}{l}\text { Sparger } \\
\text { Orifice diameter }(\mathrm{mm})\end{array}$} \\
\hline Type & Concentration & $T\left({ }^{\circ} \mathrm{C}\right)$ & & \\
\hline \multirow{3}{*}{ Distilled water } & - & 20 & Up to 14 & \multirow{3}{*}{0.3 and 0.5} \\
\hline & - & 25 & Up to 14 & \\
\hline & - & 30 & Up to 14 & \\
\hline Aqueous solutions of alcohol & Up to $3.43 \mathrm{M}$ & 25 & Up to 12 & 0.3 \\
\hline Aqueous solutions of $\mathrm{NaCl}$ & Up to $3.5 \mathrm{M}$ & 25 & Up to 12 & 0.3 \\
\hline Aqueous solutions of sucrose & Up to $1.41 \mathrm{M}$ & 25 & Up to 12 & 0.3 \\
\hline
\end{tabular}




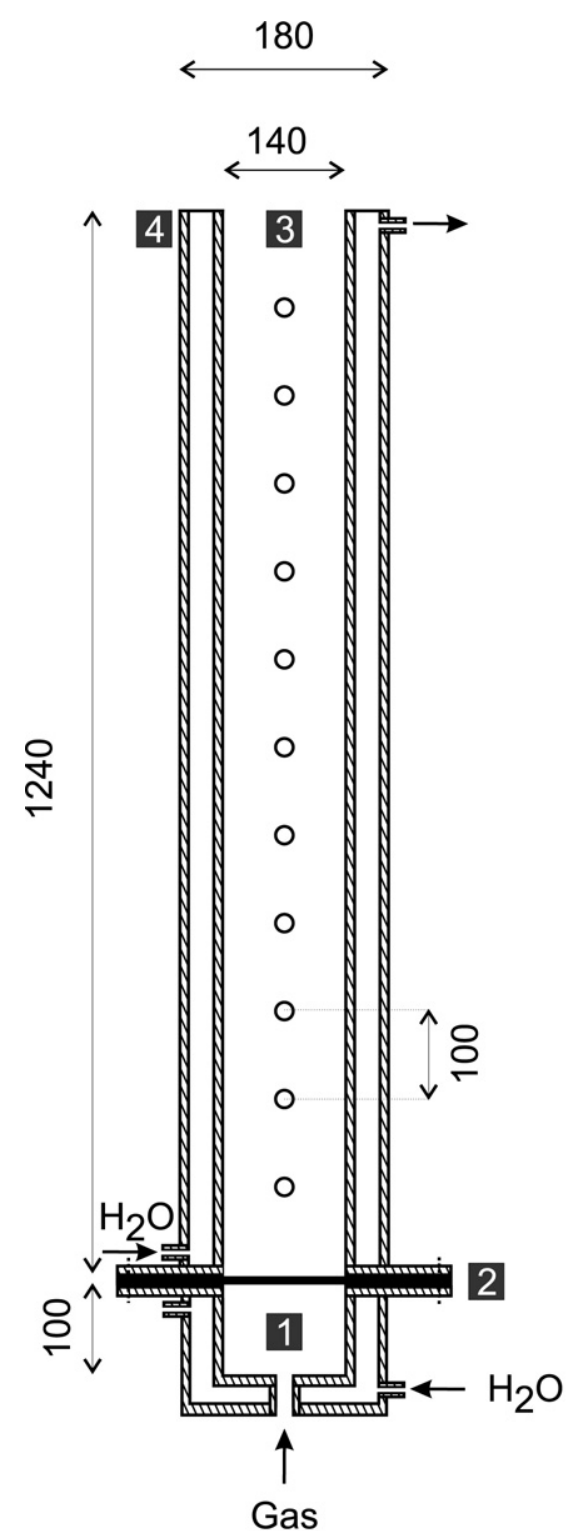

(a)

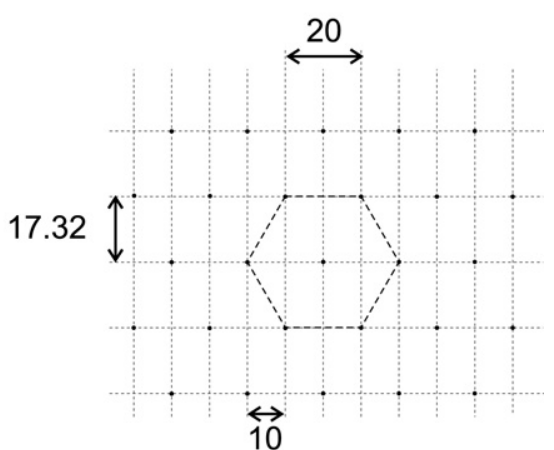

(b)

Fig. 1. Bubble column (a) and sparger (b) used in the present work (all dimensions in mm): 1 - gas chamber; 2 - sparger; 3 - cylindrical column; 4 - rectangular box.

until oxygen concentration in the liquid reaches the saturation. Dissolved oxygen concentration values were measured online using an $\mathrm{O}_{2}$ electrode (CellOx 325, WTW), located $0.20 \mathrm{~m}$ from the gas sparger and $0.07 \mathrm{~m}$ from the wall, and recorded directly in a PC, through a data acquisition board. By this way, the dissolved oxygen concentration variation with time, $t$, is obtained, and $k_{L} a$ can be calculated according to the following procedure.

The mass balance for oxygen in the liquid is written as:

$\frac{d C}{d t}=k_{L} a\left(C^{*}-C\right)$

where $C^{*}$ and $C$ are, respectively, the oxygen solubility and oxygen concentration in the liquid. Assuming the liquid phase homogeneous and $C_{0}$ the oxygen concentration at $t=0$, the integration of the previous equation leads to:

$\ln \left(C^{*}-C\right)=\ln \left(C^{*}-C_{0}\right)-k_{L} a \cdot t$.

The volumetric mass transfer coefficient can now be determined by plotting $\ln \left(C^{*}-C\right)$ against time $(t)$. The experimental results are reproducible with an average relative error of $5 \%$ and are not influenced by the dynamics of the oxygen electrode since its response time (less than $16 \mathrm{~s}$ for a $95 \%$ confidence interval) was much smaller than the mass transfer time of the system.

The solubility of oxygen in water $\left(C^{*}\right)$ was taken experimentally for each run and the slope was determined using the statistical method Test $F$. This method consists in determining the optimum number of points $\left(n_{p}\right)$ for a linear regression of the experimental data [21].

\subsection{Image analysis experiments}

\subsubsection{Set-up}

In order to obtain the bubble size distribution and, consequently, the specific interfacial area $a$, a second experimental set-up was used, Fig. 2 . The device is a perspex rectangular column $(W \times D \times H: 140 \mathrm{~mm} \times 20 \mathrm{~mm} \times 1240 \mathrm{~mm})$ covered by a perspex rectangular box to control the temperature through water circulation and to minimize the optical distortion. The column was designed in order to minimize the problems related with the effect 


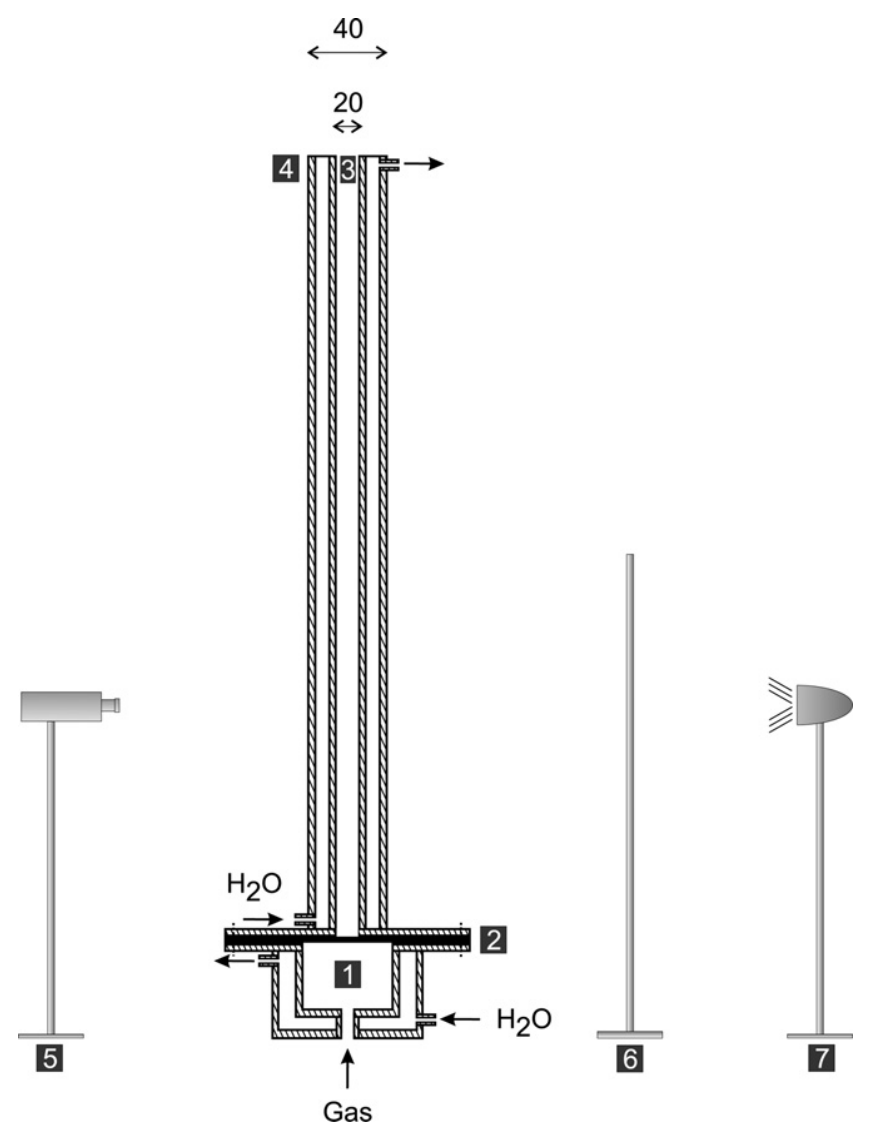

Fig. 2. Experimental set-up for visualization measurements (all dimensions in $\mathrm{mm}$ ): 1 - gas chamber; 2 - sparger; 3 - rectangular column $(W \times D \times H: 140 \mathrm{~mm} \times 20 \mathrm{~mm} \times 1240 \mathrm{~mm}) ; 4$ - rectangular box; 5 - digital camera; 6 - diffuser glass; 7 - halogen lamp.

of bubble position in the column on bubble size measurement using image analysis techniques. By this way, it was possible to reduce the error, associated to this effect, for a value less than $3 \%$. Moreover, no influence on bubble size and shape was verified using this rectangular column in comparison with the bubble column used in mass transfer experiments. A gas chamber is located at the bottom where the gas enters first and then passes through a steel sparger, with a thickness of $2 \mathrm{~mm}$, where the bubbles are formed. The sparger is constituted by a single line of orifices with a pitch of $20 \mathrm{~mm}$ and a relative free area of $0.02 \%$ and $0.05 \%$ for a orifice diameter of 0.3 and $0.5 \mathrm{~mm}$, respectively. Sets of images, obtained $0.2 \mathrm{~m}$ from the gas sparger, were grabbed with a black and white high speed digital video camera (frame rate of 250 images/s) connected to a PC, and used to study the bubble shape and size in the same conditions of $k_{L} a$ determinations. After the acquisition a set of images (about 5 images/s) are automatically treated and the bubbles are identified and classified. For that, the image analysis technique and the discriminant factorial analysis (DFA) were combined.

\subsubsection{Methodology}

- Image analysis

Bubbles images acquisition was carried out as previously referred. These images are then treated, analyzed and several numerical descriptors are extracted for each bubble or group of bubbles using Visilog $\operatorname{Tm}^{5}$ (Noesis, les Ulis, France).

The image treatment consists of: reduction of the color depth of the image from 256 levels of gray to 2 colors, hole filling, noise elimination, elimination of the objects that contact the board of the image and identification of the bubbles in the image (Fig. 3 ).

The image descriptors are projected bubble area, $A_{\text {proj }}$, from which the equivalent spherical bubble diameter $\left(D_{e q}=\right.$ $2 \sqrt{A_{\text {proj }} / \pi}$ ) is deduced, perimeter $P, N_{i}$ (number of internal zones, the central and transparent parts of the bubble), Feret diameters distribution, from which the maximal $\left(F_{\max }\right)$ and minimal $\left(F_{\min }\right)$ are deduced (Fig. 4). The Feret diameter is the distance between two parallel tangent to the silhouette and making an angle $\alpha$ with the vertical. From these parameters a set of secondary parameters are calculated: (i) circularity $C=P^{2} /\left(4 \pi A_{\text {proj }}\right.$ ); (ii) elongation $\left(F_{\max } / F_{\text {min }}\right)$; (iii) aspect ratio $\left(F_{\max } / D_{e q}\right)$; (iv) areas ratio $\left(A_{\text {proj }} / S_{\text {in }}\right)$, where $S_{\text {in }}$ is the surface occupied by the internal zones, the central and transparent part of the bubble (as an example, see the single bubbles in Fig. 5); (v) box ratio $\left(S_{\text {box }} / A_{\text {proj }}\right)$,
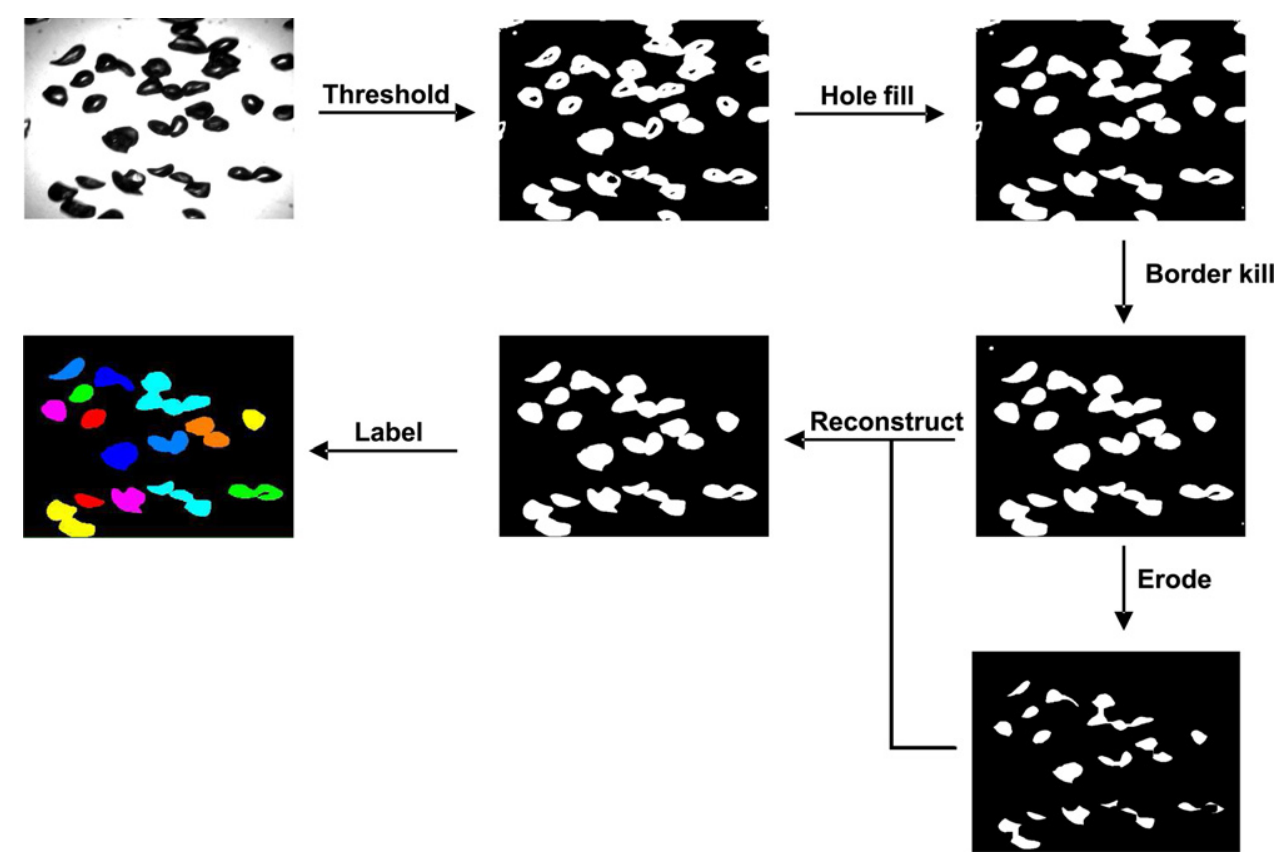

Fig. 3. Sequence of operations performed on the images before numerical descriptors extraction. 


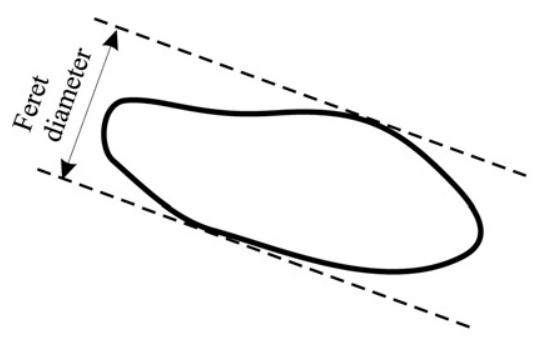

(a)

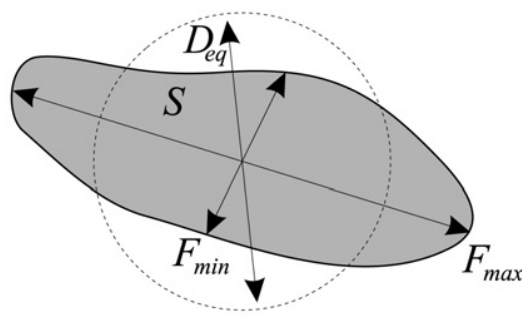

(b)

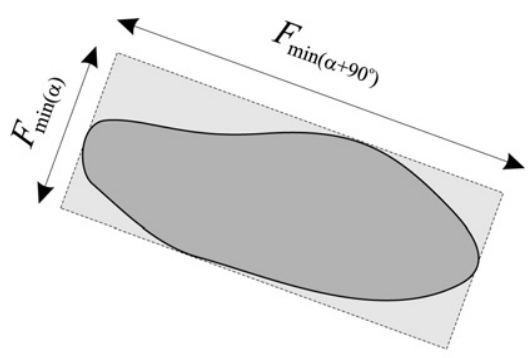

(c)

Fig. 4. Image analysis parameters. Feret diameters (a); equivalent diameter (b); $S_{b o x}$ (c).

where $S_{b o x}=F_{\min }(\alpha) \cdot F_{\min }\left(\alpha+{ }^{90^{\circ}}\right)$; (vi) the bubble robustness $\left(\Omega_{1}\right)$ $[17,22]$.

- Bubbles classification

In order to obtain an automated classification of the bubbles, the DFA method was trained, using the parameters obtained from the image analysis applied to a population test of 1000 bubbles visually classified by an operator, according to Fig. 5 . The method performs the distinction among single bubbles and bubble groups (medium complexity and large complexity groups). The trained algorithms are then used to automatically classify the bubbles in the system. A statistical toolbox, XLstat (T. Fahmy, Paris, France), running under Excel (Microsoft) was used for that purpose.

The degree of mismatch between the automated and visual classifications is evaluated by the following performance index

$P I(\%)=\left(1-\frac{\text { Number of misclassified bubbles }}{\text { Total number of bubbles }}\right) \times 100$
The previous automatic classification is based on several probabilities of each bubble belonging to each of the groups considered $[17,18]$. Based on that information it is possible to determine the complexity of the bubble system through the parameter bubble complexity degree $\left(B_{C D i}\right)$. This parameter was calculated through the following equation:

$B_{C D i}=100\left(P b_{i, S}+\frac{3}{2} P b_{i, M C}+2 P b_{i, V C}-1\right)$

where $P b_{i, S}, P b_{i, M C}$ and $P b_{i, V C}$ are the probabilities of each bubble (i) being a single bubble, or belonging to a medium complexity or large complexity bubbles group, respectively.

The mean complexity degree of the entire population $B_{C D}$ is calculated through the following equation:

$B_{C D}=\frac{\sum_{i}^{n} B_{C D i}}{n}$

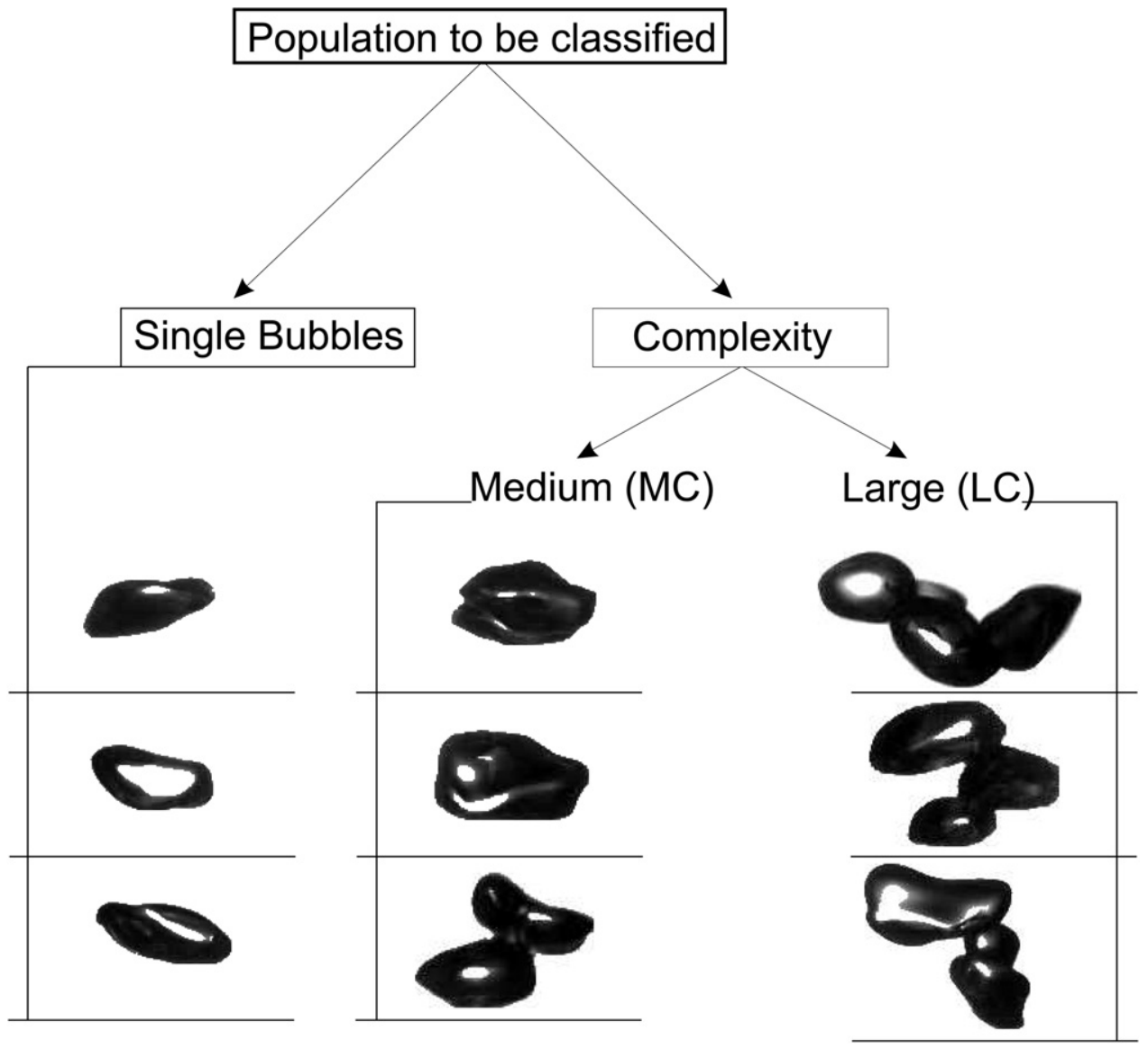

Fig. 5. Classification tree for bubble groups. 
- Bubble size distribution and interfacial area

The most important bubbles' geometric parameter for quantifying the size of each individual bubble is the equivalent diameter. As the shape of the bubbles is influenced by the superficial velocity and, usually, these present a spheroid geometry [15], this parameter was determined from the superficial area of the bubble $\left(A_{\text {sup }_{i}}\right)$ according to the following equation:

$D_{e q_{i}}=2 \sqrt{\frac{A_{s u p_{i}}}{4 \pi}}$

where

$A_{s u p_{i}}=2 \pi r_{1}^{2}+\pi r_{1} r_{2} \ln \left(\frac{r_{1}+r_{2}}{r_{1}-r_{2}}\right)$

and, $2 r_{1}$ and $2 r_{2}$ correspond to the maximal Feret diameter and minimal Feret diameter, respectively.

For processes involving mass transfer through an interfacial area, the bubble size distribution (BSD) is well represented by the Sauter mean diameter $\left(d_{32}\right)$ which is given by

$d_{32}=\frac{\sum_{i} n_{i} \cdot D_{e q_{i}}^{3}}{\sum_{i} n_{i} \cdot D_{e q_{i}}^{2}}$

The specific interfacial area, $a$, can be determined from the gas hold-up, $\varepsilon_{G}$, and the BSD as follows:

$a=6 \frac{\varepsilon_{G}}{d_{32}}$

The volume fraction of the gas phase was measured by bed expansion with a mean error less than $5 \%$.

\subsubsection{Determination of physical properties}

The surface tension was measured experimentally using the pendant drop method. For that the software SCA20 was utilized.

The viscosity, $\mu$, of the different solutions was obtained from $[23,24]$ works.

\section{Results and discussion}

\subsection{Bubble classification}

The bubble classification study begins with the automatic classification performance. The performance index obtained for the population test was as follows: single bubbles $98 \%$; medium complexity bubble group 59\%; and large complexity bubble group $76 \%$. As one can see the performance index for the distinction between single bubbles and bubbles groups is very high, showing a high agreement between the automated and visual classifications. The automatic classification of medium complexity bubble group presented seems not so good, as a result of the higher subjectivity level of this classification. The same problem was reported by Faria et al. [17], Faria [25] and Ferreira et al. [18,26].

After the bubbles classification it is now possible to obtain the correct bubble size distribution, as well as its shape, based on single bubbles measurements. In order to obtain the distribution curves with a statistical meaning, about 800 bubbles were analysed for each experimental condition. This number is in accordance with the value (500 bubbles) found by Colella et al. [12] on their literature review. The same value was obtained by Faria et al. [17] in sucrose crystals classification. According to the authors a minimum of 500 particles should be analysed to insure a deviation smaller than $5 \%$ for $D_{e q}$ and $2 \%$ for the shape descriptors and an acceptable stability of the coefficients of variation, i.e. of the distribution parameters.
The time required for the image analysis technique with statistical meaning (500 bubbles) is less than $1 \mathrm{~min}$ for bubble size measurements and about $10 \mathrm{~min}$ for population classification.

The image analysis results obtained for the different systems and experimental conditions are presented next, together with the mass transfer results for the same conditions.

\subsection{Temperature and gas sparger influence on $k_{L}$ and a}

The temperature and gas sparger influence on $k_{L}$ and $a$ was studied on air $/ \mathrm{H}_{2} \mathrm{O}$ system. Therefore, mass transfer experiments were performed using different temperatures and gas spargers (orifice diameters of 0.3 and $0.5 \mathrm{~mm}$ ), and, in same conditions, bubble sizes and gas holdups were obtained, Fig. 6(a)-(c), respectively. In our previous work [27] the temperature influence on the individual parameters of $k_{L} a$ was studied in a bubble column with an internal diameter of $84 \mathrm{~mm}$ using a gas sparger with an orifice diameter of $0.3 \mathrm{~mm}$ and the following correlation was obtained:

$k_{L} a=3.372 \times 10^{3} \sqrt{\frac{D_{L}}{\pi} \sqrt{\frac{u_{G} g}{\mu_{l}}}} u_{G}^{0.87} \mu^{*^{-0.24}}$

where $\mu^{*}$ is the effective viscosity of the particle-fluid mixture calculated based on Einstein expression [28] which linearly relates the effective viscosity and the particles concentration (three phase system) as follows:

$\frac{\mu^{*}}{\mu_{l}}=1+2.5 f$

where $\mu_{l}$ is the viscosity of the fluid and $f$ the volumetric concentration of the particles.

The previous correlation (Eq. (9)) seems to predict well the mass transfer process at different temperatures using the sparger with an orifice diameter of $0.3 \mathrm{~mm}$ (Fig. 6(a)). However, the prediction of the sparger effect on $k_{L} a$ cannot be done by this equation, that being the main reason of its fail when the sparger with an orifice diameter of $0.5 \mathrm{~mm}$ is used.

In what concerns the influence of gas sparger type on $a$, it can be concluded that different orifice diameters produce different $a$ values (Fig. 6(d)) and, it seems, also, that $a$ increases slightly with the temperature. However, as this small variation is within the experimental error (5\%), the temperature influence on $a$ is not possible to predict. The experimental values obtained using an orifice diameter of $0.5 \mathrm{~mm}$ are about $20 \%$ less compared with the ones obtained using the orifice diameter of $0.3 \mathrm{~mm}$. These deviations seem to be related with different bubble sizes produced. According to Fig. 6(b) the bubble diameter has different behaviors with the superficial gas velocity increase, when different orifice diameters of gas sparger are used. Using the image analysis technique it was possible to identify three different zones of bubble sizes. At the first one, an opposite behavior on the bubble size is observed, when different gas spargers are used. According to the results, bubble size decreases when $u_{G}$ increases, for the gas sparger with an orifice diameter of $0.5 \mathrm{~mm}$. This behavior is directly related with the number of orifices actually used for low superficial gas velocities. In these conditions the sparger starts acting as a single nozzle producing big bubbles which, according to Fig. 6(f), seem to flow not individually but as a group (the higher the $B_{C D}$ parameter, the higher the tendency of bubbles flowing in group). As a consequence the mass transfer is reduced [29]. The formation of big bubbles has two effects on hydrodynamics with an important influence on the mass transfer process. On the one hand, the interfacial area available is reduced, and a direct consequence on the mass transfer is observed. On the other hand, the bubble size increase also increases its velocity, the deformability of the bubbles and oscillation amplitudes, influencing by this way the mass transfer. Montes et al. [30] show 


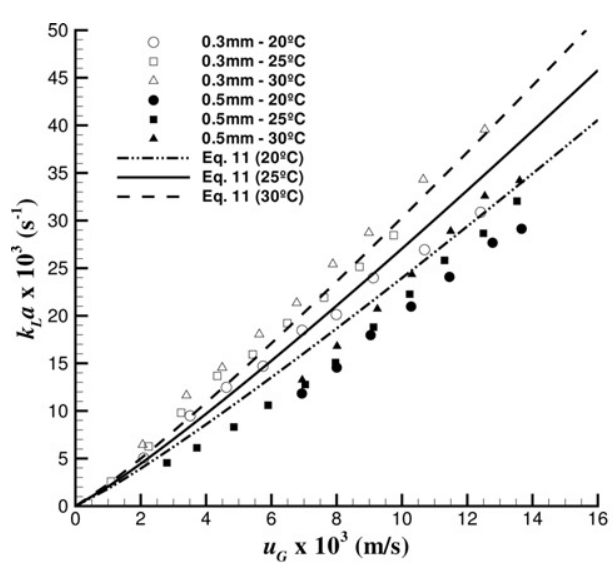

(a)

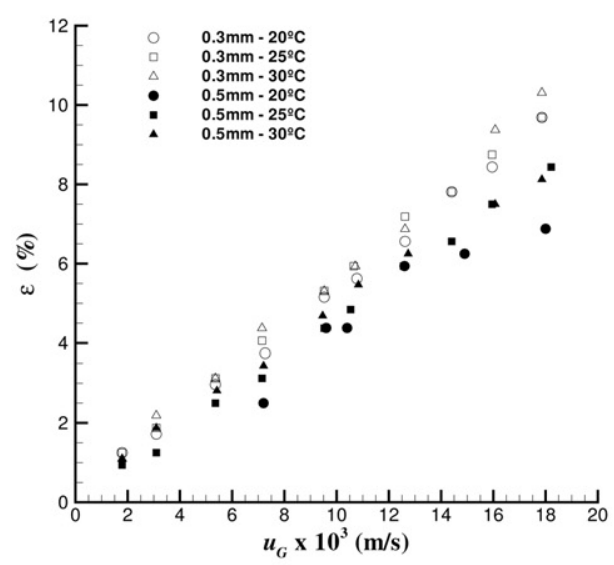

(c)

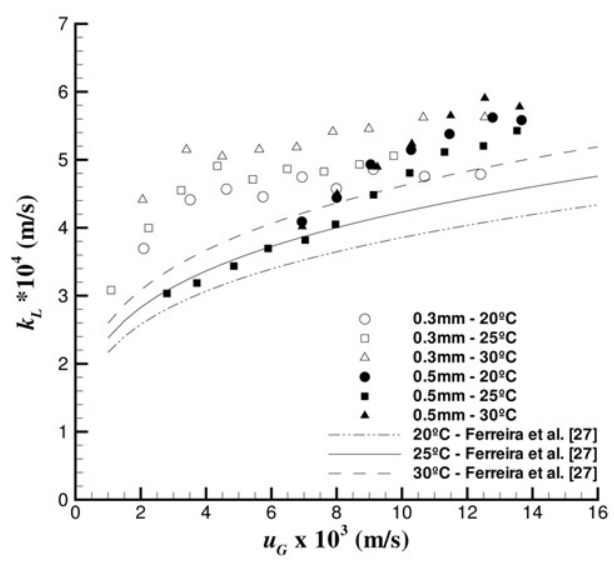

(e)

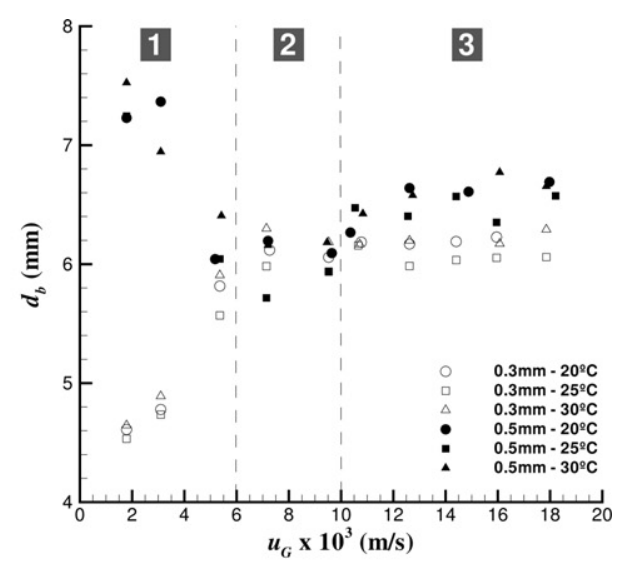

(b)

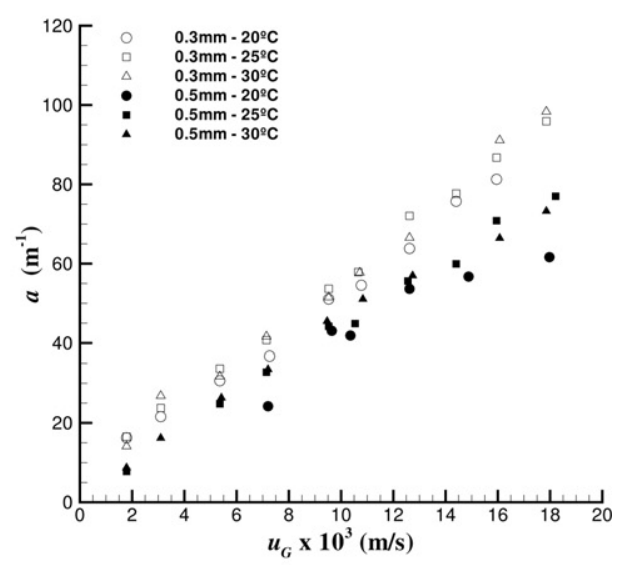

(d)

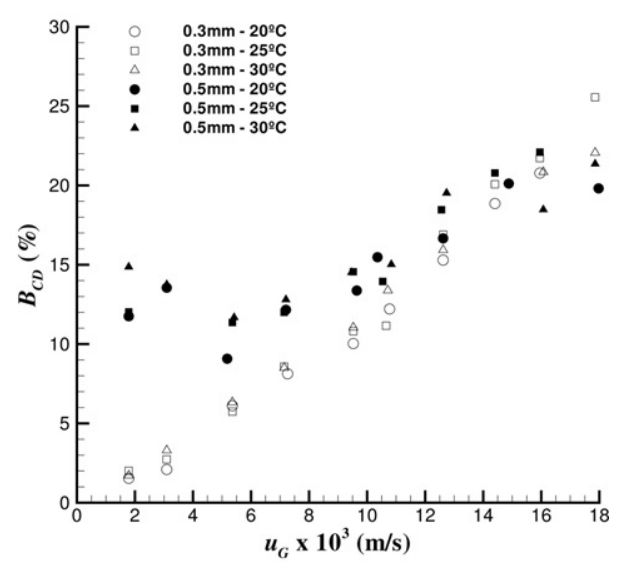

(f)

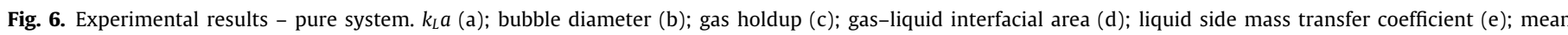
complexity degree (f).

that oscillating bubbles improve the mass transfer due to the variation of the contact times and the concentration profiles surrounding the bubbles. Martín et al. [10] found that bubbles generated in a $1.5 \mathrm{~mm}$ diameter orifice are almost rigid, while the bubbles resulting from the coalescence process are big enough to oscillate. In this case, the authors show that the superficial area reduction can be balanced with the increase of the amplitude of the oscillation. In the present work, it was observed that all bubbles present an oscillating movement, this increasing with the bubble size. However, when comparing the first zone of Fig. 6(b) for the two spargers one observes that the sparger with larger orifices produces larger bubbles with smaller $k_{L}$ values (Fig. 6(e)). The present results suggest that the phenomena reported by the previous authors seem to not have a significant influence on the present work. The results obtained for $k_{L}$ seem to indicate that $k_{L}$ obtained for one single bubble, cannot be used in a dispersion of bubbles because of the 
presence of other bubbles. Koynov et al. [19] found that in bubble swarms, bubbles no longer traveled by themselves, but rather in liquid perturbed by the wakes of neighboring bubbles. In addition, the concentration of gas dissolved in the liquid around the bubble in a swarm no longer depended only on the mass transfer from the bubble itself, but also on the mass transfer from the other bubbles in the swarm. These two factors resulted in a decrease in the mass transfer coefficient of the bubble swarm compared with a single bubble. These subjects were taken into account by Martín et al. [20] in order to study, theoretically, the bubble deformation contribution to the mass transfer. The authors found that these phenomena can explain the wide range of parameters often used to fit $k_{L}$ and the fact that in bubble columns the concentration profiles surrounding individual bubbles are not completely developed due to the presence of others bubbles. According to the previous works the influence of the other bubbles on the individual bubbles seems to be an important task on mass transfer process. In order to clarify that subject and its influence on our $k_{L}$ and $k_{L} a$ results the bubble population complexity degree $\left(B_{C D}\right)$ was obtained at different experimental conditions for the two spargers (Fig. 6(f)). Based on the new parameter, $B_{C D}$, it is possible to know if a bubble flow alone or in a bubble group. As one can see, in zones 1 and 2 (identified in Fig. 6(b)) the complexity of the bubble population is higher when the sparger with $0.5 \mathrm{~mm}$ orifice diameter is used. This indicates that the bubbles formed using the sparger with $0.5 \mathrm{~mm}$ orifice diameter flow, mostly, in a bubble group. Therefore, and according to the literature, the $k_{L}$ values obtained for the large bubbles seem to be justified as a result of the influence of the other bubbles on the concentration profiles surrounding the individual bubbles. From Fig. 6(b), (e) and (f) one can infer that:

- Systems with different bubble population complexity degrees, $B_{C D}$, present (zones 1 and 2 ) different $k_{L}$ values, the highest being the ones with the lowest $B_{C D}$ values. $k_{L}$, in these conditions, seems to be bubble size independent;

- Systems with similar bubble population complexity degrees and different bubble sizes (zone 3 ) present $k_{L}$ bubble size dependent.

\subsection{Viscosity and superficial tension influence on $k_{L}$ and $a$}

The liquid viscosity and surface tension effects on hydrodynamics and bubble characteristics have been investigated in bubble columns over the years [14,16,29,31-34]. However, the relation of these physical properties with the individual components of volumetric liquid side mass transfer coefficient, mainly with $k_{L}$, remains poorly studied. In the present work the image analysis technique was used to study the effect of the mentioned liquid properties on $a$. From $a$ and $k_{L} a$ results the quantification of $k_{L}$ is possible. This study was performed using aqueous solutions of alcohol, $\mathrm{NaCl}$ and sucrose with different concentrations (Table 1 ), and began with the experimental determination of surface tension. Fig. 7 shows the comparison of experimental and literature values [35-38]. A good agreement was obtained, the observed differences seeming to be probably related to the type of water used, as a systematic deviation was observed. The viscosities of the different solutions were obtained from Chenlo et al. [23] work and Simmonds [24] book, and are presented in Fig. 8. As one can see the sucrose has the biggest effect on the solution viscosity.

Fig. 9 presents characteristic images of pure (only water) and impure (aqueous solutions of alcohol $(1.37 \mathrm{M}), \mathrm{NaCl}(2 \mathrm{M})$ and sucrose $(1.41 \mathrm{M})$ ) systems as well as the bubble size distributions obtained. As one can see, the bubble size decreases for impure systems, the sucrose system being the one that presents the higher quantity of small bubbles (less than $0.8 \mathrm{~mm}$ in diameter). The bimodal distributions obtained for impure systems seem to be associated to the liquid properties. On the one hand, according to Mouza

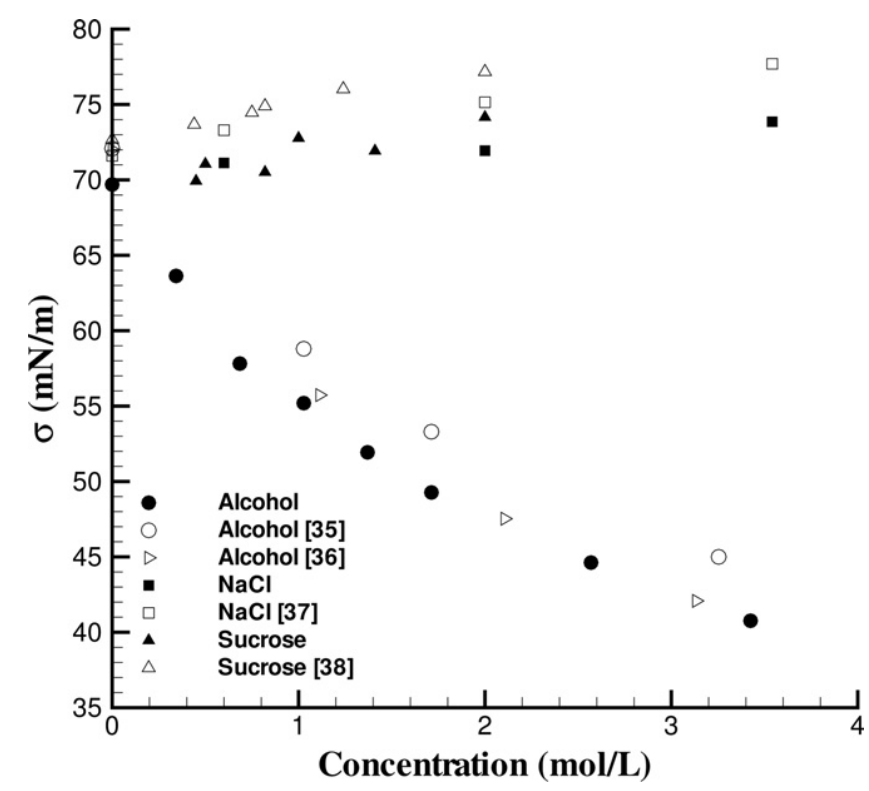

Fig. 7. Surface tension of the different solutions (experimental: • - Alcohol, $\mathbf{\square}-$ $\mathrm{NaCl}$, and $\mathbf{\Delta}$ - Sucrose; literature: $\circ-$ Vásquez et al. [35], $\triangleright-$ Kiriyanenko et al. [36], $\square-$ Vanhanen et al. [37] and $\triangle-$ Honig [38]).

et al. [16] at relatively low viscosity (less than $8.2 \mathrm{cP}$ ) an increase in viscosity hinders film drainage during the thinning process and thus inhibits coalescence. On the other hand, a decrease in liquid surface tension favors small bubble formation by promoting breakage and demoting coalescence. Moreover, the superficial gas velocity also plays an important role on bubble size, shape and population complexity (Fig. 10). From Fig. 10(a) and (b) the differences in the bubble sizes, when they are determined using the mean bubble size $\left(d_{10}\right)$ or the Sauter mean diameter, become evident. These deviations are related with the small bubbles production when the air/water system is contaminated. In what concerns the bubble shape, this is related to the bubble size. Observing Fig. 10(a) and (c) it seems that liquid properties have some effect on bubble shape namely at low superficial velocities. Thus, globally, the impurities decrease the bubble size, induce the small bubbles formation and the elongation reduction. In what concerns the bubble population complexity (Fig. 10(d)), the differences are observed for

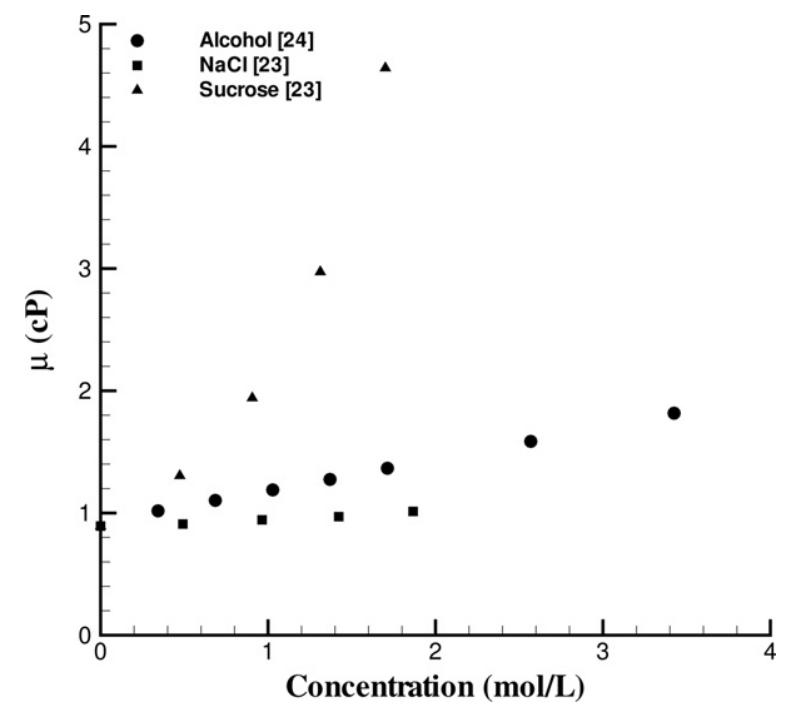

Fig. 8. Viscosity of the different solutions obtained from the literature: Chenlo et al. [23] ( $\square$ and $\Delta$ ) and Simmonds [24] (•). 


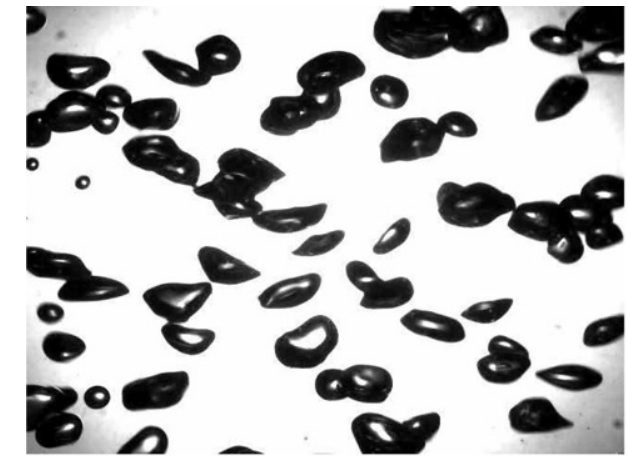

(a)

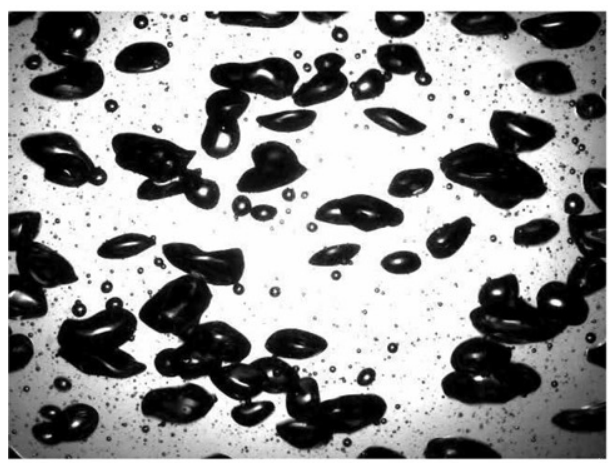

(c)

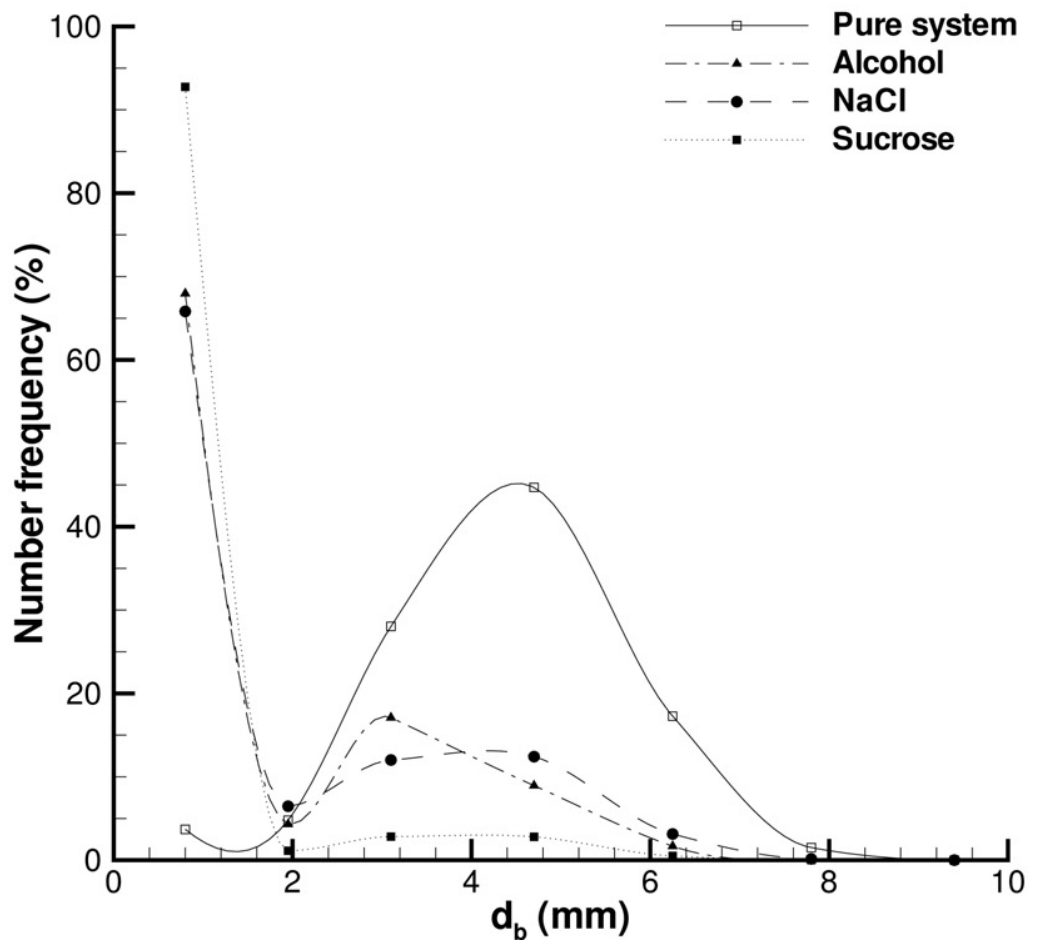

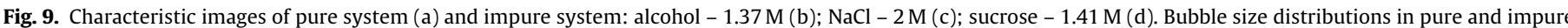
systems $\left(u_{G}=14 \times 10^{-3} \mathrm{~m} / \mathrm{s}, 25^{\circ} \mathrm{C}\right.$, sparger: $0.3 \mathrm{~mm}$ orifice diameter $)(\mathrm{e})$.

$u_{G}>10 \times 10^{-3} \mathrm{~m} / \mathrm{s}$. It seems, in these conditions, that alcohol and $\mathrm{NaCl}$ decrease the bubble population complexity, decreasing, by this way, the influence of others bubbles on the mass transfer of a single bubble.

The impurity influence on $a$ is shown in Fig. 11 for the different impurity concentrations. As a systematic deviation of $a$ for pure

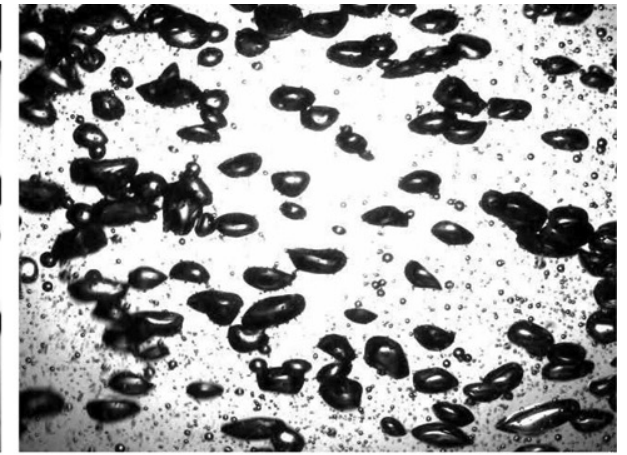

(b)

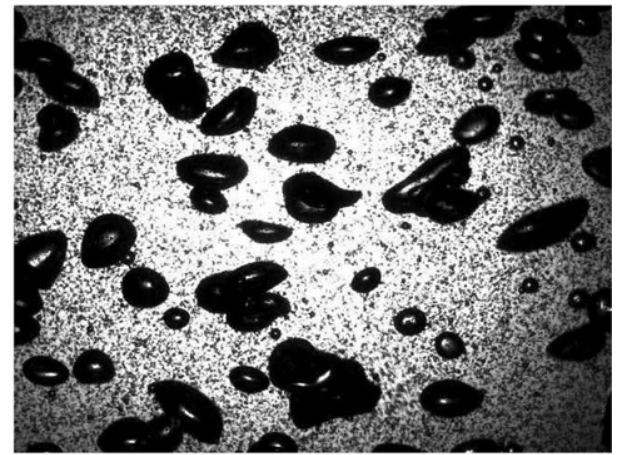

(d)

Pure system

$\mathrm{NaCl}$ 


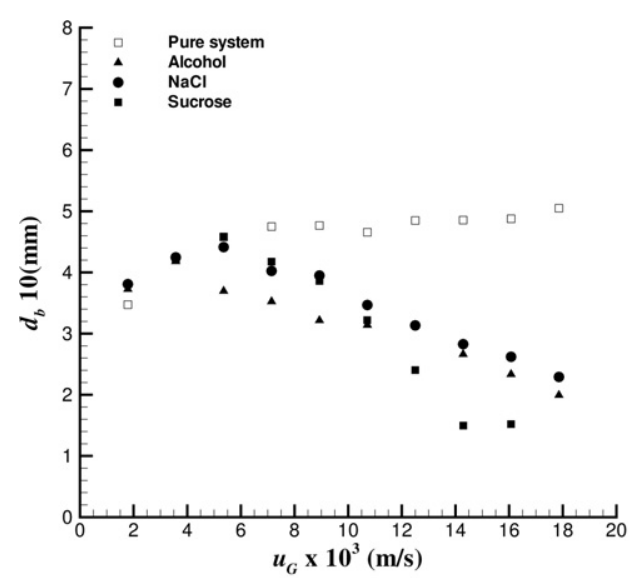

(a)

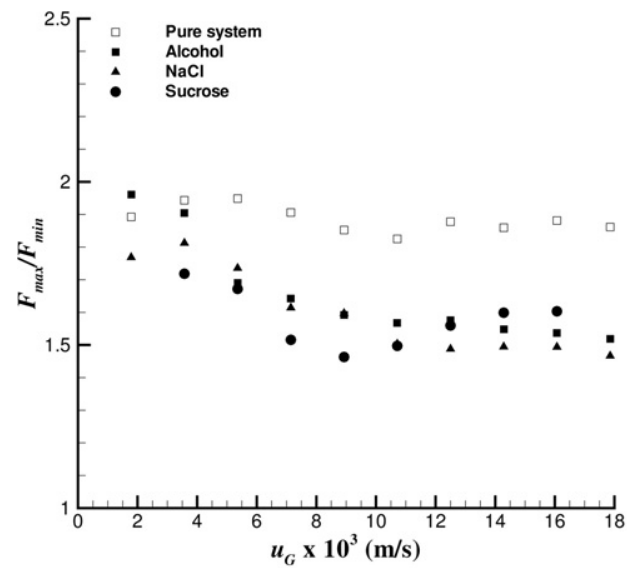

(c)

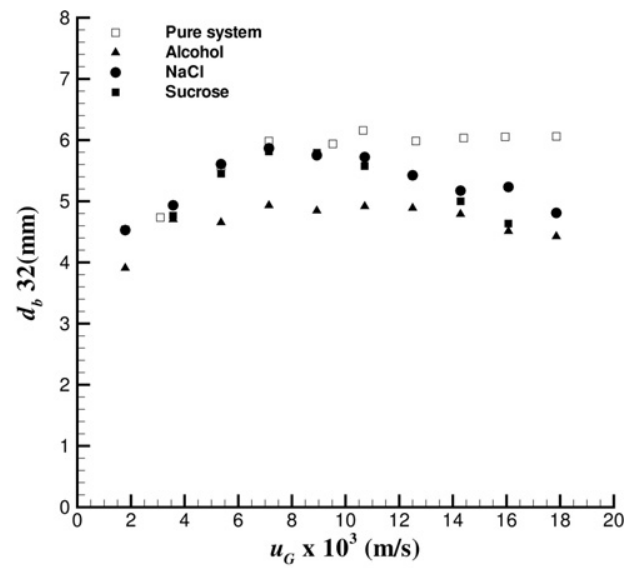

(b)

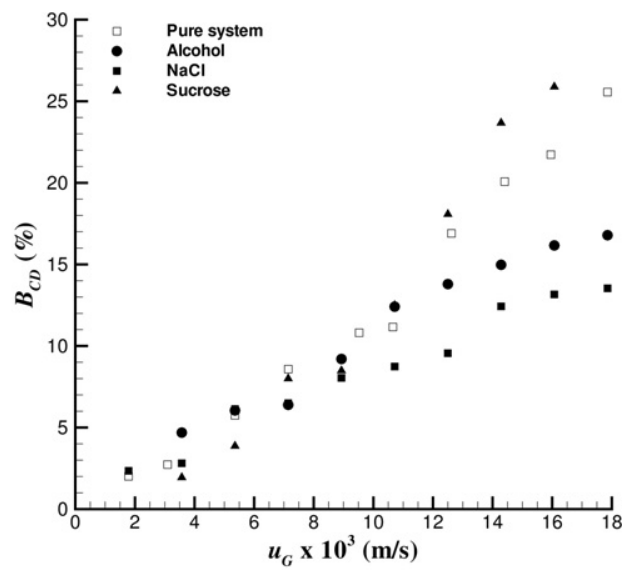

(d)

Fig. 10. Image analysis results - alcohol (1.37 M); $\mathrm{NaCl}(2 \mathrm{M})$; sucrose (1.41 M).

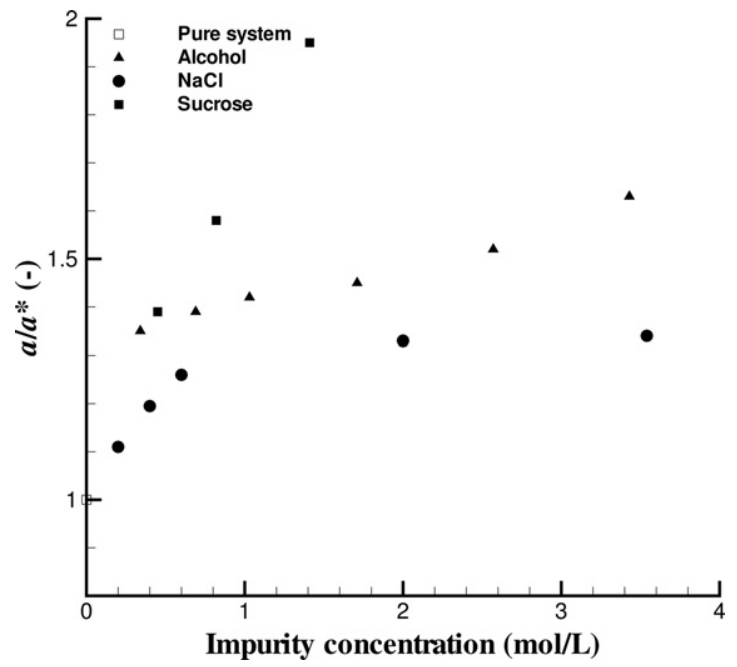

Fig. 11. Interfacial area variation for impure systems.

Table 2

Interfacial area variation with surface tension and viscosity.

\begin{tabular}{lllll}
\hline Impurity & Concentration $(\mathrm{M})$ & $\sigma(\mathrm{mN} / \mathrm{m})$ & $\mu(\mathrm{cP})$ & $a / a^{*}$ \\
\hline Sucrose & 0.82 & 70.50 & 1.90 & 1.58 \\
Alcohol & 3.43 & 40.46 & 1.82 & 1.63 \\
Sucrose & 1.41 & 71.93 & 3.33 & 1.95 \\
$\mathrm{NaCl}$ & 2.00 & 71.94 & 1.02 & 1.33 \\
\hline
\end{tabular}

observed. However, care need to be taken for a final conclusion, as different magnitudes of viscosity and surface tension are used. From Table 2 we can conclude that a $42 \%$ reduction of surface tension leads to an increase of $3 \%$ of the interfacial area (practically unchanged). On the other hand, a 330\% increase of viscosity leads to a $46 \%$ interfacial area increase. Even taking into account the differences of the relative changes on the surface tension and viscosity, it is clear that viscosity has a more decisive influence on $a$, for the experimental conditions used. This conclusion was not completely unexpected, taking into account the bubble size distribution reported previously. However, Akita et al. [31] and Mouza et al. [16] present correlations, in which the surface tension has a higher influence on $a$ than viscosity. Maceira et al. [14] report a similar effect of the both parameters. All these correlations when compared with the results of the present work, underestimate $a$.

With $a$ previously determined, $k_{L}$ can now be calculated from $k_{L} a$ values. In Fig. 12, the experimental $k_{L} a$ and $k_{L}$ values obtained for air/water/impurity systems are presented. As one can see, all impurities decrease $k_{L}$, even when an increase on $k_{L} a$ is observed (Fig. 12(a)). Ethanol is known to reduce the mean bubble size by inhibiting bubble coalescence, since the surface tension decreases $[39,40]$. Thus, it is expected an $a$ increase with ethanol concentration. This was observed in the present work (Fig. 11). As the increase in $k_{L} a$ is not very significant, consequently, alcohol will affect $k_{L}$ decreasing it (Fig. 12(b)). This parameter maintains almost unchanged with alcohol concentration variation. In $\mathrm{NaCl}$ systems an increase in the impurity concentration favors $k_{L}$ decrease. As 


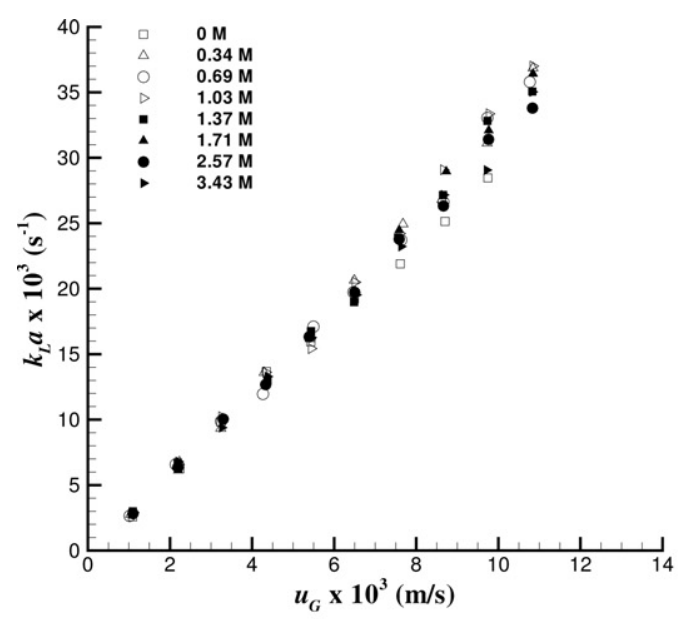

(a)

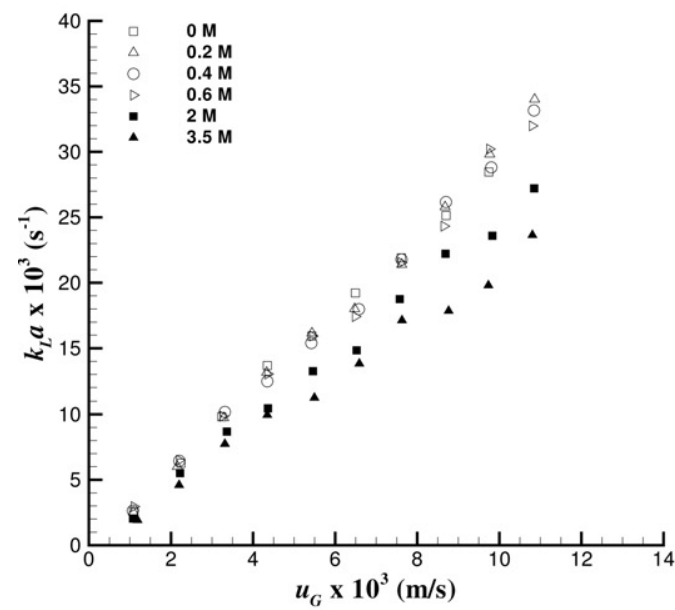

(c)

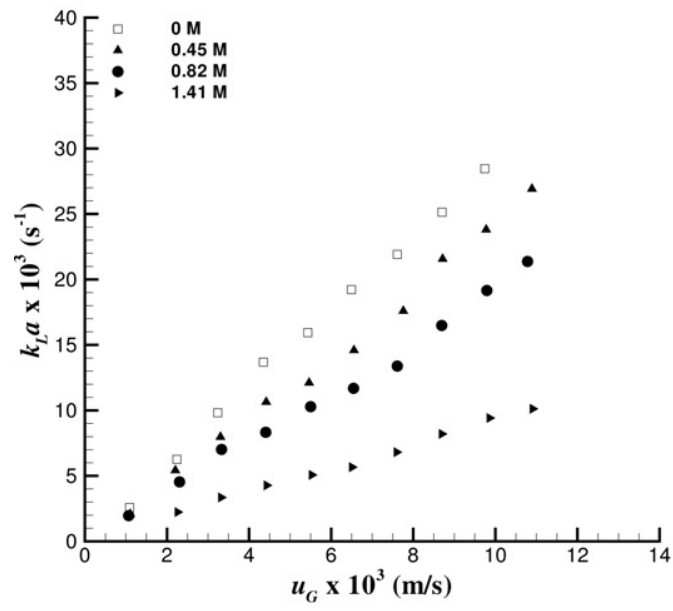

(e)

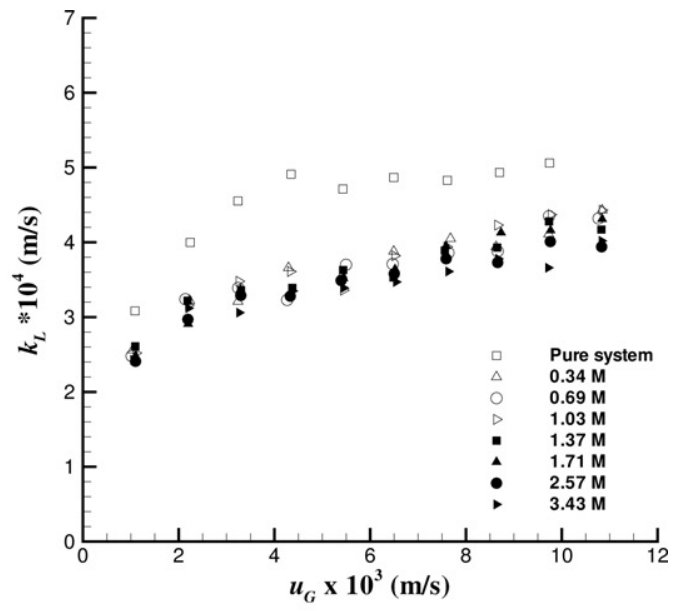

(b)

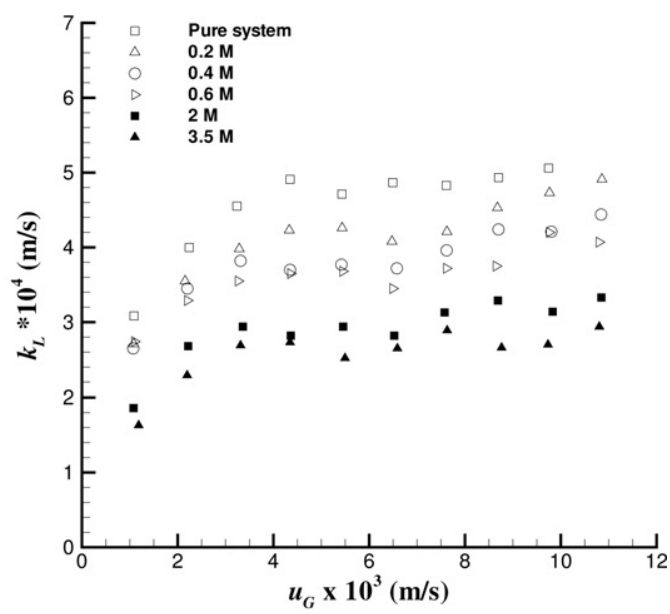

(d)

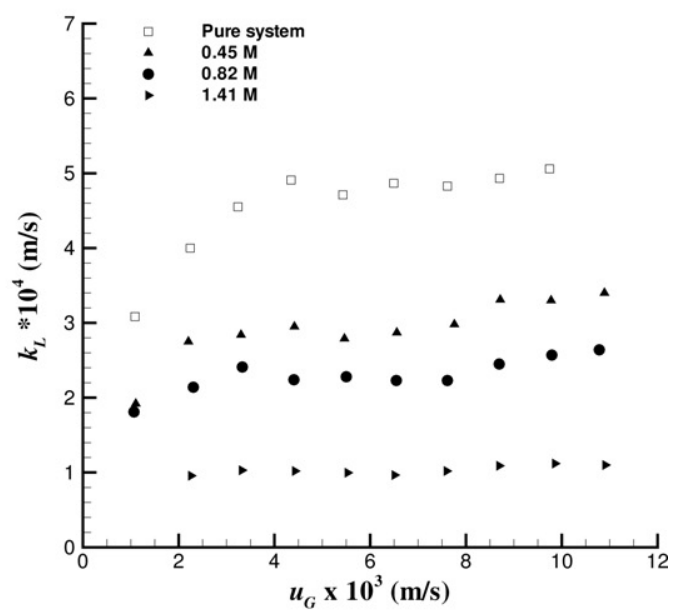

(f)

Fig. 12. Dependence of $k_{L} a$ and $k_{L}$ on superficial gas velocity for impure systems. Alcohol (a) and (b); $\mathrm{NaCl}$ (c) and (d); sucrose (e) and (f).

already referred an increase in $a$ is observed, this result being in agreement with Orvalho et al. [41] work regarding to the electrolytes effect on bubble column hydrodynamics. The biggest influence on $k_{L} a$, as well as on $k_{L}$, is observed when sucrose is used.
As $a$ increases with the impurity concentration, sucrose decreases significantly the liquid-side mass transfer coefficient, probably acting on the oxygen molecular diffusion. According to Martín et al. [42] the liquid viscosity has two effects on the hydrodynamics of 


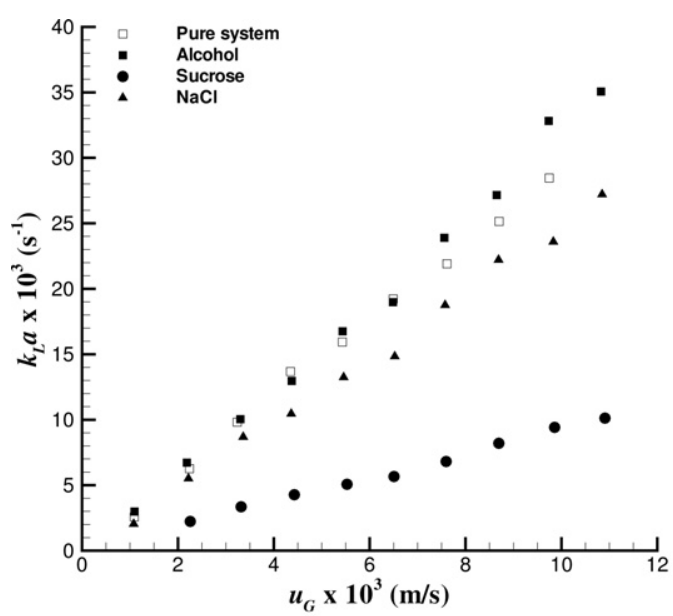

(a)

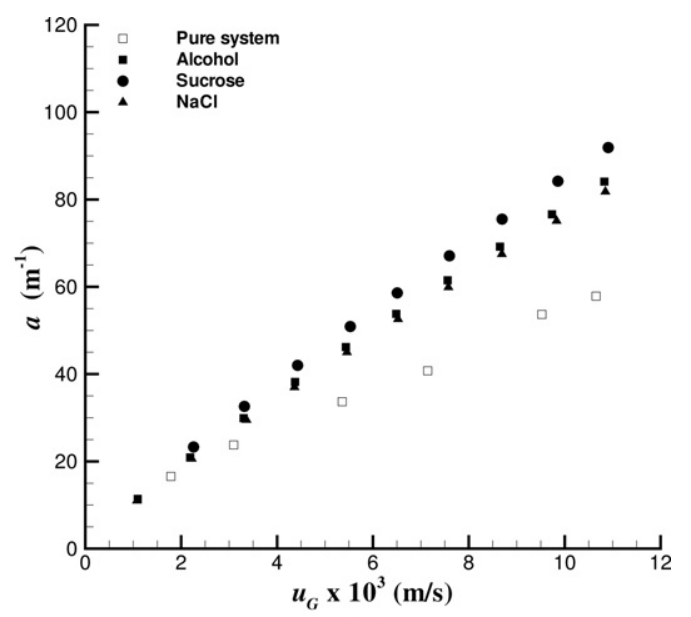

(b)

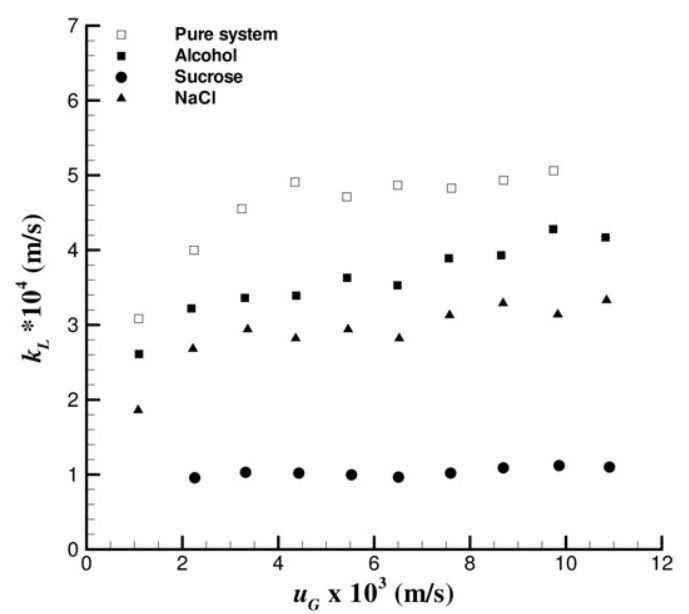

(c)

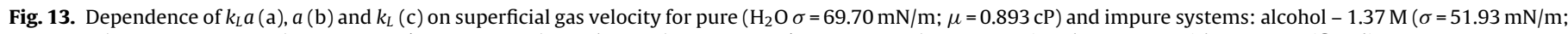
$\mu=1.28 \mathrm{cP})$; sucrose $-1.41 \mathrm{M}(\sigma=71.93 \mathrm{mN} / \mathrm{m} ; \mu=3.33 \mathrm{cP})$; $\mathrm{NaCl}-2 \mathrm{M}(\sigma=71.94 \mathrm{mN} / \mathrm{m} ; \mu=1.02 \mathrm{cP})$, at $25^{\circ} \mathrm{C}$ using the sparger with $0.3 \mathrm{~mm}$ orifice diameter.

the bubble. First, it slows down the bubble expansion, and second, it slows down the molecular movement at the surface of the bubble. The retardation of the molecular movement reduces the diffusivity and, consequently, $k_{L}$. The presented results are also in agreement with Ozturk et al. [43] work.

In short, the impurities affect the specific interfacial area, increasing it, and decrease $k_{L}$, these effects being more or less pronounced. To analyze separately, the effects of surface tension and viscosity, Fig. 13 was built. In this figure, results that refer to different experimental conditions are presented: with the same (or very approximate) surface tension and different viscosity, or the same viscosity and different surface tension. $\mathrm{H}_{2} \mathrm{O}, \mathrm{NaCl}(2 \mathrm{M})$ and sucrose $(1.41 \mathrm{M}$ ) present similar surface tension (around $70 \mathrm{mN} / \mathrm{m}$ ) and different viscosities; $\mathrm{H}_{2} \mathrm{O}, \mathrm{NaCl}(2 \mathrm{M})$ and alcohol $(1.37 \mathrm{M})$ present similar viscosity (around $1 \mathrm{cP}$, approximately) and different surface tensions.

From Fig. 13(a) one observes that the viscosity has a big impact on $k_{L} a$. The effect is mainly on $k_{L}$ as it is seen comparing Fig. 13(b) and (c). Comparing the systems with the same viscosity and different surface tension (pure and $\mathrm{NaCl}$ systems with alcohol system) one does not find so significant differences as in the previous case. In what concerns $k_{L} a$ the differences are small. Further, observing Fig. 13(b) and (c) one finds that systems $\left(\mathrm{H}_{2} \mathrm{O}\right.$ and $\left.\mathrm{NaCl}\right)$ with the same viscosity and the same surface tension present different results, showing, by this way that other variable(s) can be responsible for this phenomenon. As the effects on $k_{L}$ and $a$ are opposite they partially compensate and so the effect in $k_{L} a$ is small as referred before. Further experiments using different ionic species need to be performed for a final conclusion regarding the salts effect on the individual parameters of $k_{L} a$.

\section{Conclusions}

The image analysis tool, developed in the present work, was successfully used for automatic identification of single bubbles and bubble groups in a bubble column. The agreement between the automated and visual classification was about $90 \%$, being the performance index of the single bubbles of $98 \%$. When different orifice diameters of gas sparger are used, bubble size behaviors differently with superficial gas velocity $\left(u_{G}\right)$ increase. When the gas sparger with $0.5 \mathrm{~mm}$ orifice diameter is used a $20 \%$ reduction in interfacial area is observed, when compared with the results obtained using the $0.3 \mathrm{~mm}$ orifice diameter.

It seems that surface tension has a smaller influence on specific interfacial area when compared with viscosity effect. The viscosity appears to have a considerable influence on $a$ and $k_{L}$. Temperature seems to act mainly on $k_{L}$ variation as no significant changes on $a$ were observed. With the image analysis technique it was also possible to quantify the complexity level of a bubble population at 
different mass transfer conditions. This information was very useful for a better knowledge of hydrodynamic phenomena on mass transfer process. According to the results, $k_{L}$ values obtained for the different experimental conditions are also justified as a result of the influence of the other bubbles on the concentration profiles surrounding the individual bubbles.

\section{Acknowledgement}

This work was supported by Fundação para a Ciência e Tecnologia under program contract number SFRH/BPD/45637/2008.

\section{References}

[1] Y.T. Shah, B.G. Kelkar, S.P. Godbole, W.D. Deckwer, Design parameters estimations for bubble column reactors, AIChE J. 28 (1982) 353-379.

[2] E. Sada, H. Kumazawa, C. Lee, N. Fujiwara, Gas-liquid mass transfer characteristics in a bubble column with suspended sparingly soluble fine particles, Ind. Eng. Chem. Proc. Des. Dev. 24 (1985) 255-261.

[3] E. Alper, W.D. Deckwer, P.V. Danckwerts, Comparison of effective interfacial areas with the actual contact area for gas absortion in a stirred cell, Chem. Eng. Sci. 35 (1980) 1263-1268.

[4] G. Quicker, A. Schumpe, W.D. Deckwer, Gas-liquid interfacial areas in a bubble column with suspended solids, Chem. Eng. Sci. 39 (1984) 179-183.

[5] F. Camacho, E. Molina, F. Valdés, J.M. Andujar, Influence of operating and physical variables on interfacial area determination, AIChE J. 37 (1991) 1196-1204.

[6] G. Vázquez, M.A. Cancela, C. Reverol, E. Alvarez, J.M. Navaza, Determination of interfacial areas in a bubble column by different chemical methods, Ind. Eng. Chem. Res. 39 (2000) 2541-2547.

[7] G. Vázquez, M.A. Cancela, C. Riverol, E. Alvarez, J.M. Navaza, Application of the Dankwerts method in a bubble column. Effect of surfactants on mass transfer coefficient and interfacial area, Chem. Eng. J. 78 (2000) 13-19.

[8] C. Boyer, A.-M. Duquenne, G. Wild, Measuring techniques in gas-liquid and gas-liquid-solid reactors, Chem. Eng. Sci. 57 (2002) 3185-3215

[9] M. Martín, F.J. Montes, M.A. Galán, Bubbling process in stirred tank reactors. II. Agitator effect on the mass transfer rates, Chem. Eng. Sci. 63 (12) (2008) 3223-3234.

[10] M. Martín, F.J. Montes, M.A. Galán, Bubble coalescence at sieve plates. II. Effect of coalescence on mass transfer. Superficial area versus bubble oscillations, Chem. Eng. Sci. 62 (6) (2007) 1741-1752.

[11] Y.H. Tsang, Y.-H. Koh, D.L. Koch, Bubble-size dependence of the critical electrolyte concentration for inhibition of coalescence, J. Colloid Interface Sci. 275 (1) (2004) 290-297.

[12] D. Colella, D. Vinci, R. Bagatin, M. Masi, E.A. Bakr, A study on coalescence and breakage mechanisms in three different bubble columns, Chem. Eng. Sci. 54 (21) (1999) 4767-4777.

[13] M.J. Prince, H.W. Blanch, Bubble coalescence and break-up in air-sparged bubble columns, AIChE J. 36 (10) (1990) 1485-1499.

[14] R. Maceiras, E. Álvarez, M. Cancela, Experimental interfacial area measurements in a bubble column, Chem. Eng. J. 163 (3) (2010) 331-336.

[15] P.C. Mena, M.C. Pons, J.A. Teixeira, F.A. Rocha, Using image analysis in the study of multiphase gas absorption, Chem. Eng. Sci. 60 (2005) 5144-5150.

[16] A.A. Mouza, G.K. Dalakoglou, S.V. Paras, Effect of liquid properties on the performance of bubble column reactors with fine pore spargers, Chem. Eng. Sci. 60 (2005) 1465-1475.

[17] N. Faria, M.N. Pons, S. Feyo de Azevedo, F.A. Rocha, H. Vivier, Quantification of the morphology of sucrose crystals by image analysis, Powder Technol. 133 (2003) 54-67.
[18] A. Ferreira, N. Faria, F. Rocha, J. Teixeira, Using an online image analysis technique to characterize sucrose crystal morphology during a crystallization run, Ind. Eng. Chem. Res. 50 (11) (2011) 6990-7002.

[19] A. Koynov, J.G. Khinast, G. Tryggvason, Mass transfer and chemical reactions in bubble swarms with dynamic interfaces, AIChE J. 51 (10) (2005) 2786-2800.

[20] M. Martín, F.J. Montes, M.A. Galán, Mass transfer from oscillating bubbles in bubble column reactors, Chem. Eng. J. 151 (1-3) (2009) 79-88.

[21] P. Mena, A. Ferreira, J. Teixeira, F. Rocha, Effect of some solid properties on gas-liquid mass transfer in a bubble column, Chem. Eng. Process. 50 (2)(2011) $181-188$.

[22] M.N. Pons, H. Vivier, J. Dodds, Particle shape characterization using morphological descriptors, Part. Part. Syst. Charact. 14 (1997) 272-277.

[23] F. Chenlo, R. Moreira, G. Pereira, A. Ampudia, Viscosities of aqueous solutions of sucrose and sodium chloride of interest in osmotic dehydration processes, J. Food Eng. 54 (4) (2002) 347-352.

[24] C. Simmonds, Alcohol, Its Production, Properties, Chemistry, and Industrial Applications, Macmillan \& Co., 1919.

[25] N. Faria, Quantificaç ao da morfologia de cristais de açúcar e sua aplicaç ao ao estudo das cinéticas de cristalizaç ao, Ph.D. thesis, Faculdade de Engenharia da Universidade do Porto, Rua Dr. Roberto Frias, s/n 4200-465 Porto, Portugal, 2001.

[26] A. Ferreira, N. Faria, F. Rocha, S. Feyo de Azevedo, A. Lopes, Using image analysis to look into the effect of impurity concentration in $\mathrm{NaCl}$ crystallization, Chem. Eng. Res. Des. 83 (A4) (2005) 331-338.

[27] A. Ferreira, C. Ferreira, J.A. Teixeira, F. Rocha, Temperature and solid properties effects on gas-liquid mass transfer, Chem. Eng. J. 162 (2) (2010) 743-752.

[28] A. Einstein, A new determination of molecular dimensions, Ann. Phys. 19(1906) 289-306.

[29] W.D. Deckwer, Bubble Column Reactors, J. Wiley, Chichester, 1992.

[30] F. Montes, M. Galan, R. Cerro, Mass transfer from oscillating bubbles in bioreactors, Chem. Eng. Sci. 54 (15-16) (1999) 3127-3136.

[31] K. Akita, F. Yoshida, Bublle size, interfacial area, and liquid-phase mass transfer coefficient in bubble columns, Ind. Eng. Chem. Proc. Des. Dev. 13 (1974) 84-91.

[32] Y. Kawase, B. Halard, M. Moo-Young, Theoretical prediction of volumetric mass transfer coefficients in bubble columns for Newtonian and non-Newtonian fluids, Chem. Eng. Sci. (1987) 1609-1617

[33] H. Hikita, S. Asal, H. Kikukawa, T. Zalke, M. Ohue, Heat transfer coefficient in bubble column, Ind. Eng. Chem. Proc. Des. Dev. 20 (1981) 540-545.

[34] K. Koide, A. Takazawa, M. Komura, H. Matsunaga, Gas holdup and volumetric liquid-phase mass transfer coefficient in solid-suspended bubble columns, J. Chem. Eng. Jpn. 17 (1984) 459.

[35] G. Vásquez, E. Alvarez, J.M. Navaza, Surface tension of alcohol + water from 20 to $50^{\circ} \mathrm{C}$, J. Chem. Eng. Data 40 (1995) 611-614.

[36] A.A. Kiriyanenko, A.N. Solovév, Measurement of the surface tension of alcohol-water solutions by the "two-jump" method, Zhurnal Prikladnoi Mekhaniki i Tekhnicheskoi Fiziki 9 (1968) 145-148.

[37] J. Vanhanen, A.-P. HyvArinen, T. Anttila, T. Raatikainen, Y. Viisanen, H Lihavainen, Ternary solution of sodium chloride, succinic acid and water; surface tension and its influence on cloud droplet activation, Atmos. Chem. Phys. 8 (16) (2008) 4595-4604.

[38] P. Honig, Principles of Sugar Technology, vol. 1, Elsevier, 1953.

[39] S. Wachi, A. Jones, T. Elson, Flow dynamics in a drafttube bubble column using various liquids, Chem. Eng. Sci. 46 (1991) 657-663.

[40] C. Freitas, J.A. Teixeira, Effect of liquid-phase surface tension on hydrodynamics of a three-phase airlift reactor with an enlarged degassing zone, Bioprocess Eng. 19 (1998) 451-457.

[41] S. Orvalho, M.C. Ruzicka, J. Drahos, Bubble column with electrolytes: gas holdup and flow regimes, Ind. Eng. Chem. Res. 48 (17) (2009) 8237-8243.

[42] M. Martín, F.J. Montes, M.A. Galán, Oxygen transfer from growing bubbles: effect of the physical properties of the liquid, Chem. Eng.J. 128 (1)(2007)21-32.

[43] S.S. Ozturk, A. Schumpe, W.-D. Deckwer, Organic liquids in a bubble column: holdups and mass transfer coefficients, AIChE J. 33 (9) (1987) 1473-1480. 